\title{
STRUCTURAL MODIFICATION OF LIGNAN COMPOUNDS ISOLATED FROM NECTANDRA SPECIES (LAURACEAE)
}

\author{
VÍCTOR MACÍAS-VILLAMIZAR ${ }^{1, I^{*},}$, LUÍS CUCA-SUÁREZ ${ }^{1}$ \\ ${ }^{1}$ Departamento de Química, Laboratorio de Productos Naturales, Universidad Nacional de Colombia. Av. Carrera 30 \# 45-03. Cód. postal 111321; Edificio \\ 476-Oficina 11; Bogotá D.C., Colombia. §Profesor Universidad del Magdalena, Colombia.
}

\section{1-INTRODUCTION}

In the genus Nectandra, the presence of certain types of secondary metabolites has been determined, including sesquiterpenes, phytosterols, polyalcohols, arylpropionic acid derivatives, flavonols, arylpropanoids, furofuran lignans, dihydrobenzofuran neolignans [1], and certain norlignans [2], alkaloids [3], tannins [4], diterpenes [5], and components of essential oils [6]. However, the chemotaxonomic characteristics are determined by the presence of lignan-type compounds [7]. The ultimate goal of structural modification of natural products is to obtain new drugs [8]. In that sense, there is a growing interest in lignans and their synthetic derivatives due to applications in cancer chemotherapy and various other pharmacological effects [9]. This work corresponds to the first report of this type of structural modification of lignan compounds (7,7'-epoxylignans and diaryldimethylbutane lignans) isolated from Nectandra species. Therefore, this work can be used as a starting point for structure-activity relationship studies.

\section{EXPERIMENTAL}

\section{Materials and reagents}

Benzyl bromide (Merck), acetone and toluene were freshly distilled before use. 2,3-Dichloro-5,6-dicyano-1,4-benzoquinone (DDQ) (Fluka purum ,97\%), 1,4-dioxane (Aldrich, anhydrous 99.8\%), pyridine (ACS reagent, $\geq 99.0 \%$, Sigma-Aldrich) and acetic anhydride (ACS reagent, $\geq 98.0 \%$, Sigma-Aldrich) were used. Purification of the products was carried out on a short silica gel column (100-200 mesh, Merck) using increasing percentage of ethyl acetate in hexane as elutant. NMR spectra: were recorded on a Bruker Avance 400 spectrometer $\left({ }^{1} \mathrm{H} 400 \mathrm{MHz},{ }^{13} \mathrm{C} 100 \mathrm{MHz}\right)$ using TMS as internal standard, in deuterated chloroform $\left(\mathrm{CDCl}_{3}\right)$ as solvent. The product ethers were characterized by comparing spectral data of known compounds described in the literature and analysis of the spectral data.

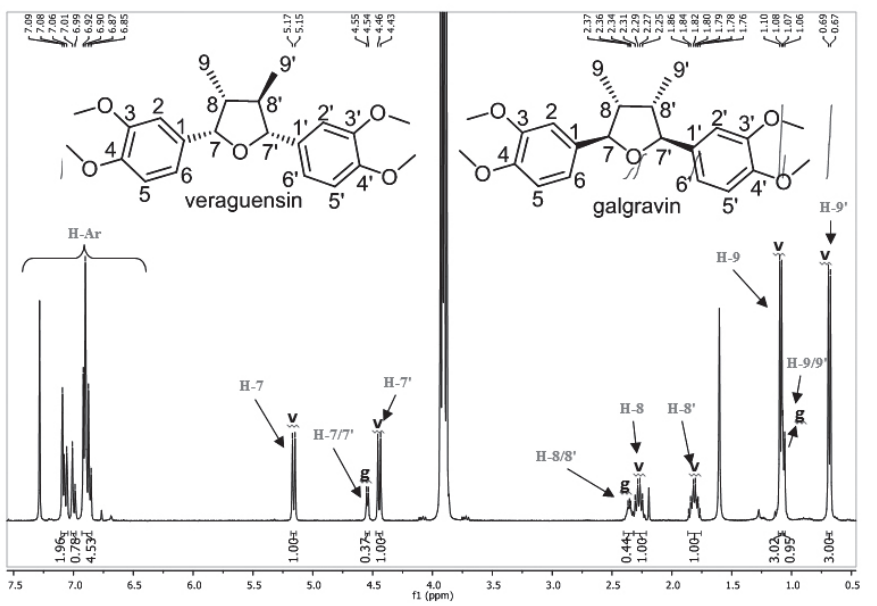

Figure 1. ${ }^{1} \mathrm{H} \mathrm{NMR}\left(400 \mathrm{MHz}, \mathrm{CDCl}_{3}\right.$ ) Spectrum of Compound 1 (mixture of veraguensin/galgravin)

Veraguensin: ${ }^{1} \mathrm{H}$ NMR (400 MHz, $\left.\mathrm{CDCl}_{3}\right), \delta: 0.67(3 \mathrm{H}, d, J=7.0, \mathrm{H}-9$ '), $1.07(3 \mathrm{H}, d, J=6.6, \mathrm{H}-9), 1.79(1 \mathrm{H}, m, \mathrm{H}-8), 2.25\left(1 \mathrm{H}, m, \mathrm{H}-8^{\prime}\right), 3.86(s$, $\left.\mathrm{OCH}_{3}\right), 3.88\left(s, \mathrm{OCH}_{3}\right), 3.89\left(s, \mathrm{OCH}_{3}\right), 3.91\left(s, \mathrm{OCH}_{3}\right), 4.42(1 \mathrm{H}, d, J=9.3$, $\mathrm{H}-7), 5.14\left(1 \mathrm{H}, d, J=8.6, \mathrm{H}-7^{\prime}\right), 6.86-7.08\left(6 \mathrm{H}, m, \mathrm{H}-2 / 5 / 6, \mathrm{H}-2^{\prime} / 5^{\prime} / 6^{\prime}\right)$. $[\mathbf{v}=$ veraguensin $]$.

Galgravin: ${ }^{1} \mathrm{H} \mathrm{NMR}\left(400 \mathrm{MHz}, \mathrm{CDCl}_{3}\right), \delta: 1.05(6 \mathrm{H}, d, J=6.7, \mathrm{H}-9 / 9$ '), $2.34\left(2 \mathrm{H}, m, \mathrm{H}-8 / 8^{\prime}\right), 3.87\left(s, \mathrm{OCH}_{3}\right), 3.88\left(s, \mathrm{OCH}_{3}\right), 4.52(2 \mathrm{H}, d, J=6.4$, $\left.\mathrm{H}-7 / 7^{\prime}\right), 6.85-6.99\left(6 \mathrm{H}, m, \mathrm{H}-2 / 5 / 6, \mathrm{H}-2^{\prime} / 5^{\prime} / 6^{\prime}\right)$. [g=galgravin].

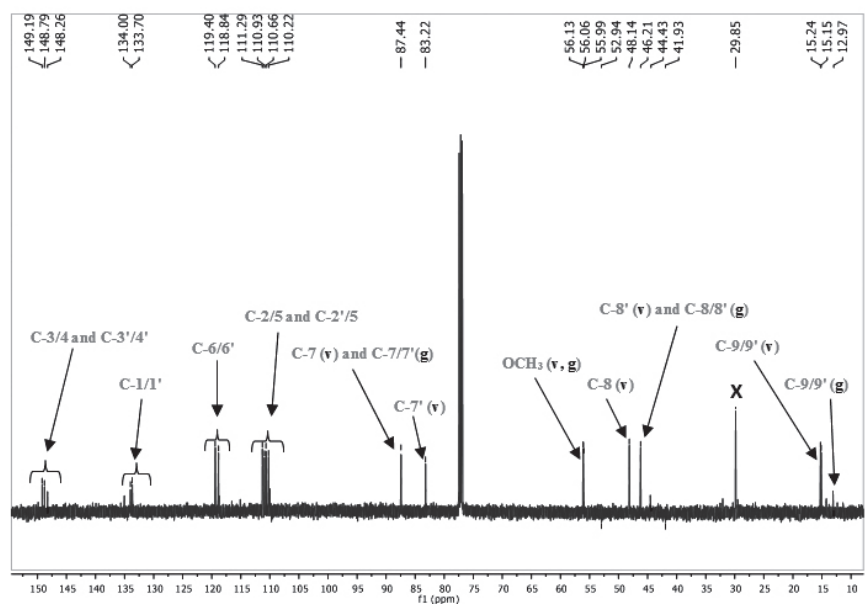

Figure 2. ${ }^{13} \mathrm{C} \mathrm{NMR}\left(100 \mathrm{MHz}, \mathrm{CDCl}_{3}\right.$ ) Spectrum of Compound $\mathbf{1}$ (mixture of veraguensin/galgravin)

Veraguensin: ${ }^{13} \mathrm{C}$ NMR (100 MHz, $\left.\mathrm{CDCl}_{3}\right) \delta: 149.1(\mathrm{C}), 148.7(\mathrm{C}), 148.7$ (C), $148.2(\mathrm{C}), 133.9(\mathrm{C}), 133.6(\mathrm{C}), 119.3(\mathrm{CH}), 118.8(\mathrm{CH}), 111.1(\mathrm{CH})$, $110.8(\mathrm{CH}), 110.5(\mathrm{CH}), 110.1(\mathrm{CH}), 87.4(\mathrm{CH}), 83.1(\mathrm{CH}), 56.1\left(\mathrm{CH}_{3}\right), 56.0$ $\left(\mathrm{CH}_{3}\right)(\mathrm{x} 2), 55.9\left(\mathrm{CH}_{3}\right), 48.0(\mathrm{CH}), 46.1(\mathrm{CH}), 15.2\left(\mathrm{CH}_{3}\right), 15.1\left(\mathrm{CH}_{3}\right)$.

Galgravin: ${ }^{13} \mathrm{C}$ NMR $(100 \mathrm{MHz}) \delta: 12.9\left(\mathrm{CH}_{3}\right), 44.3\left(\mathrm{CHCH}_{3}\right), 55.8$ $\left(\mathrm{OCH}_{3}\right), 55.9\left(\mathrm{OCH}_{3}\right), 87.2(\mathrm{OCH}(\mathrm{Ar})), 109.7$ (Ar-C2), 110.9 ( $\left.\mathrm{Ar}-\mathrm{C} 5\right), 118.5$ (Ar-C6), 134.8 (Ar-C1), 148.4 (Ar-C4), 148.9 (Ar-C3).

\section{Extraction of Secondary Metabolites}

Secondary metabolites were previously isolated from species of Nectandra $s p$. in the Natural Products Laboratory of the National University of Colombia and correspond to 7,7'-epoxilignan and diaryldimethylbutane lignans.

\section{General Procedure}

\section{Aromatization of veraguensina and galgravin (1).}

A solution of veraguensin and galgravin (90/10) 1, (5,0 mmol) and DDQ $(15,0 \mathrm{mmol})$ in toluene $(20 \mathrm{~mL})$ (the reaction mixture immediately turned deep green) was refluxed $\left(100^{\circ} \mathrm{C}\right)$ for $6 \mathrm{~h}$. The mixture was cooled, the precipitate collected, the solvent evaporated under reduced pressure, and the resulting residue purified by flash chromatography on silica gel (n-hexane/AcOEt $=7 / 3$ ) to give 2,5-bis(3,4-dimethoxyphenyl)-3,4-dimethylfuran (45\%) (TL-1) [10]. 
${ }^{l} \mathrm{HNMR}$ (400 MHz, $\mathrm{CDCl}$ ) spectral data of Veraguensin: $\delta: 0.67(3 \mathrm{H}, d, J$ $\left.=7.0, \mathrm{H}^{\prime} 9^{\prime}\right), 1.07(3 \mathrm{H}, d, J=6.6, \mathrm{H}-9), 1.79(1 \mathrm{H}, m, \mathrm{H}-8), 2.25\left(1 \mathrm{H}, m, \mathrm{H}-8^{\prime}\right)$, $3.86\left(s, \mathrm{OCH}_{3}\right), 3.88\left(s, \mathrm{OCH}_{3}\right), 3.89\left(s, \mathrm{OCH}_{3}\right), 3.91\left(s, \mathrm{OCH}_{3}\right), 4.42(1 \mathrm{H}, d, J$ $=9.3, \mathrm{H}-7), 5.14\left(1 \mathrm{H}, d, J=8.6, \mathrm{H}-7^{\prime}\right), 6.86-7.08\left(6 \mathrm{H}, m, \mathrm{H}-2 / 5 / 6, \mathrm{H}-2^{\prime} / 5^{\prime} / 6^{\prime}\right)$.

${ }^{\prime} \mathrm{HNMR}(400 \mathrm{MHz}, \mathrm{CDCl})$ spectral data of Galgravin: $\delta: 1.05(6 \mathrm{H}, d, J=$ 6.7, $\left.\mathrm{H}-9 / 9^{\prime}\right), 2.34\left(2 \mathrm{H}, m, \mathrm{H}-8 / 8^{\prime}\right), 3.87\left(s, \mathrm{OCH}_{3}\right), 3.88\left(s, \mathrm{OCH}_{3}\right), 4.52(2 \mathrm{H}, d$, $\left.J=6.4, \mathrm{H}-7 / 7^{\prime}\right), 6.85-6.99\left(6 \mathrm{H}, m, \mathrm{H}-2 / 5 / 6, \mathrm{H}-2^{\prime} / 5^{\prime} / 6^{\prime}\right)$.

H NMR (400 MHz, CDCl) spectral data of TL-1: $\delta 7.21(4 \mathrm{H}, d d, J=6.1$, $1.9, \mathrm{H}-2 / 2^{\prime}$ and $\left.\mathrm{H}-6 / 6^{\prime}\right), 6.94\left(2 \mathrm{H}, d, J=8.9, \mathrm{H}-5 / 5^{\prime}\right), 3.95\left(3 \mathrm{H}, s, \mathrm{OCH}_{3}-3 / 3^{\prime}\right)$, $3.92\left(3 \mathrm{H}, s, \mathrm{OCH}_{3}-4 / 4^{\prime}\right), 2.22\left(s, 6 \mathrm{H}, \mathrm{H}-9 / 9^{\prime}\right)$ (see supporting information, Figure 3).

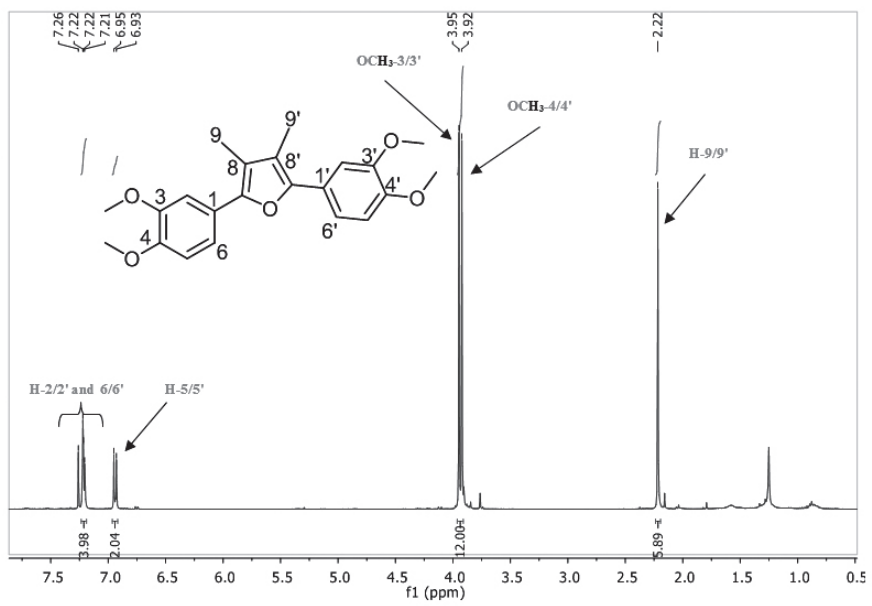

Figure 3. ${ }^{1} \mathrm{H}$ NMR ( $400 \mathrm{MHz}, \mathrm{CDCl}_{3}$ ) Spectrum of Compound 1 modified (TL-1) (mixture of veraguensin/galgravin modified)

${ }^{1} \mathrm{H}$ NMR $\left(400 \mathrm{MHz}, \mathrm{CDCl}_{3}\right) \delta 7.21(4 \mathrm{H}, d d, J=6.1,1.9, \mathrm{H}-2 / 2$ ' and $\left.\mathrm{H}-6 / 6^{\prime}\right), 6.94\left(2 \mathrm{H}, d, J=8.9, \mathrm{H}-5 / 5^{\prime}\right), 3.95\left(6 \mathrm{H}, s, \mathrm{OCH}_{3}-3 / 3^{\prime}\right), 3.92(6 \mathrm{H}, s$, $\left.\mathrm{OCH}_{3}-4 / 4^{\prime}\right), 2.22\left(6 \mathrm{H}, s, \mathrm{H}-9 / 9^{\prime}\right)$.

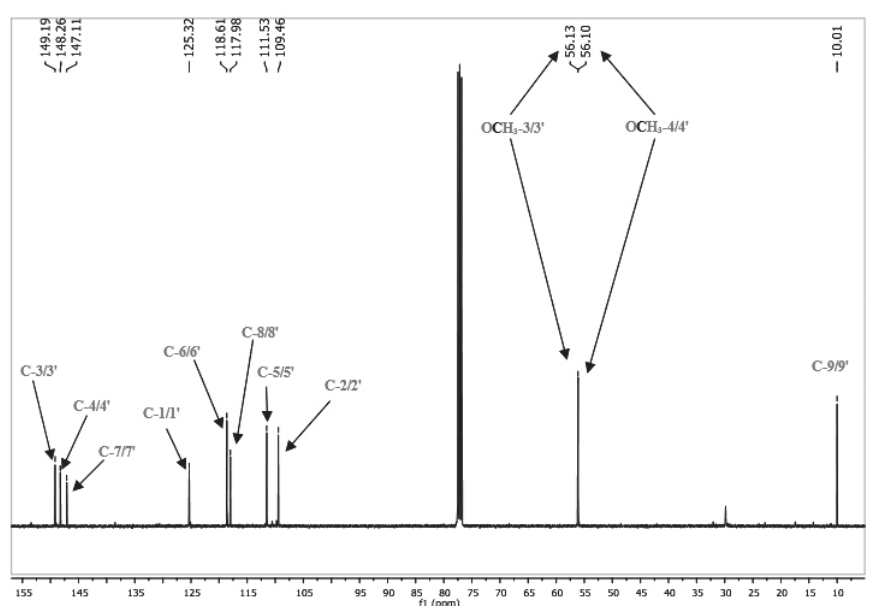

Figure 4. ${ }^{13} \mathrm{C}$ NMR ( $400 \mathrm{MHz}, \mathrm{CDCl}_{3}$ ) Spectrum of Compound 1 modified (TL-1) (mixture of veraguensin/galgravin modified)

${ }^{13} \mathrm{C}$ NMR (100 MHz, $\left.\mathrm{CDCl}_{3}\right) \delta 149.2$ (C) (x2), 148.3 (C) (x2), 147.1 (C) $(\mathrm{x} 2), 125.3(\mathrm{C})(\mathrm{x} 2), 118.6(\mathrm{CH})(\mathrm{x} 2), 117.9$ (C) (x2), $111.5(\mathrm{CH})(\mathrm{x} 2), 109.5$ $(\mathrm{CH})(\mathrm{x} 2), 56.2\left(\mathrm{CH}_{3}\right), 56.1\left(\mathrm{CH}_{3}\right), 10.0\left(\mathrm{CH}_{3}\right)(\mathrm{x} 2)$.

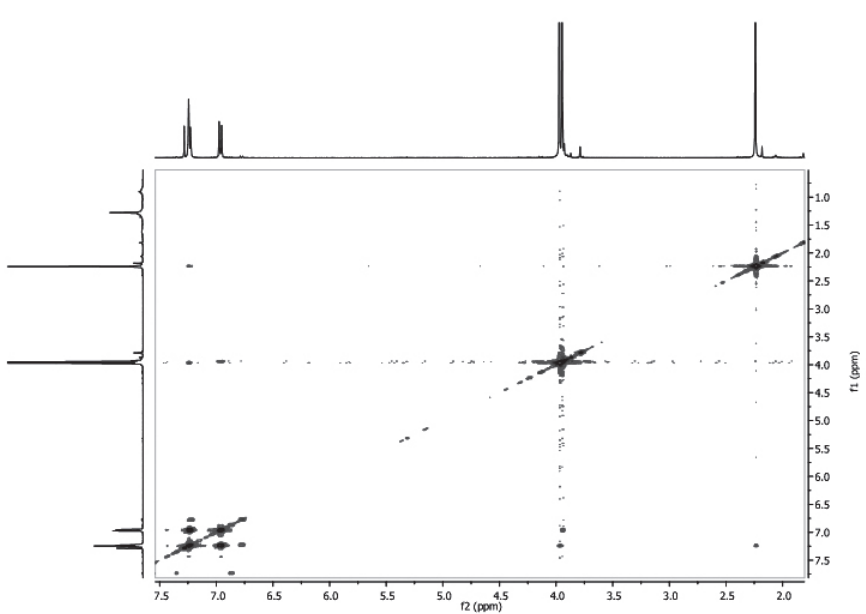

Figure 5. COSY Spectrum of Compound 1 modified (TL-1) (mixture of veraguensin/galgravin modified)

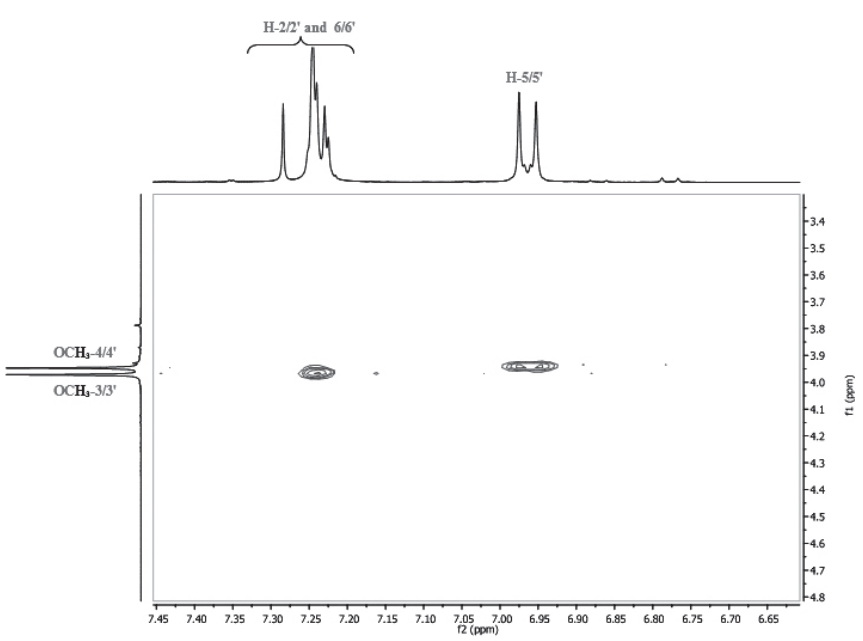

Figure 6. Expansion of the COSY Spectrum of Compound 1 modified (TL-1) (From 4.80 to $3.40_{\mathrm{f} 1}$ and From 7.45 to $6.65_{\mathrm{f} 2}$ ) (mixture of veraguensin/ galgravin modified)

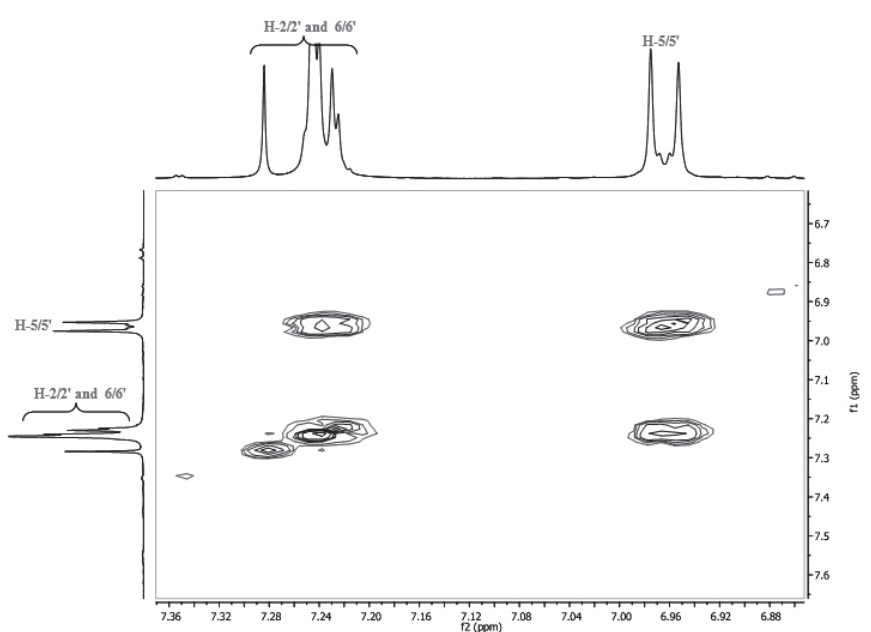

Figure 7. Expansion of the COSY Spectrum of Compound 1 modified (TL-1) (From 7.60 to $6.70_{\mathrm{fl} 1}$ and From 7.36 to $6.88_{\mathrm{f} 2}$ ) (mixture of veraguensin/ galgravin modified) 


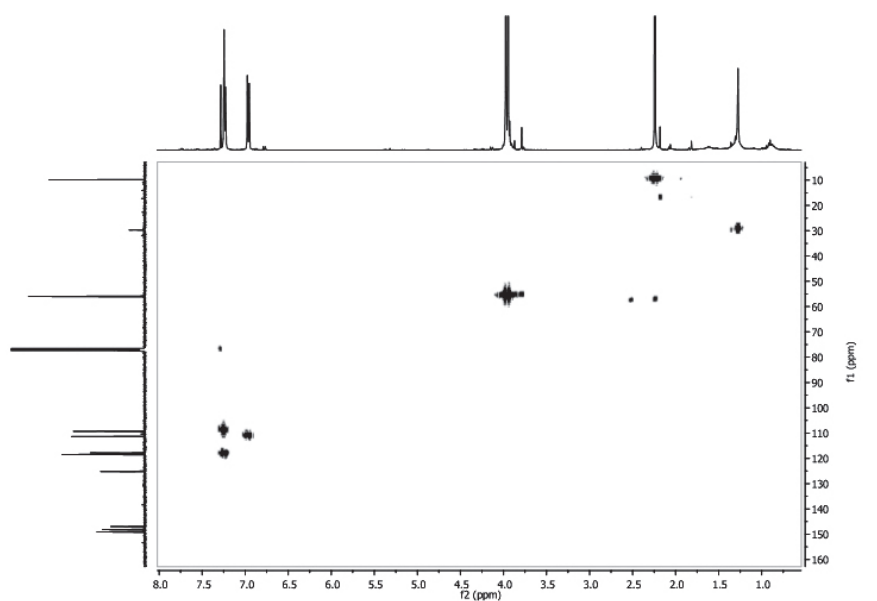

Figure 8. HMQC Spectrum of Compound 1 modified (TL-1) (mixture of veraguensin/galgravin modified)

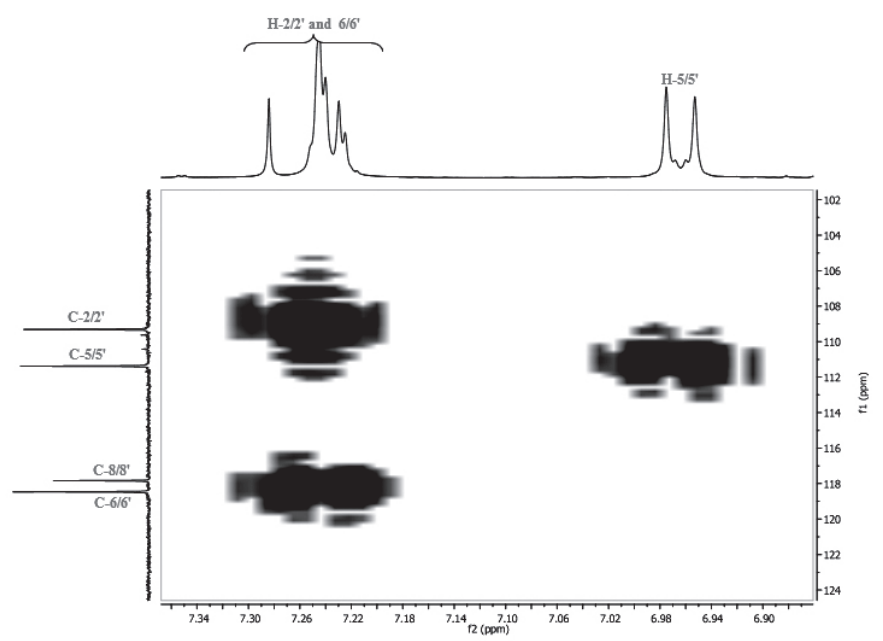

Figure 9. Expansion of the HMQC Spectrum of Compound 1 modified (TL-1) (From 124.0 to 102.0 and From 7.34 to 6.90 ) (mixture of veraguensin/galgravin modified)

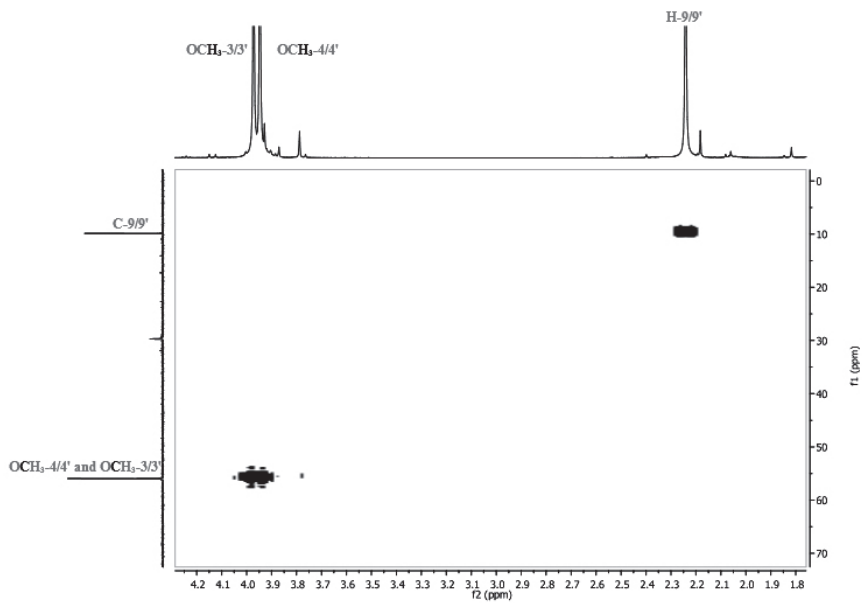

Figure 10. Expansion of the HMQC Spectrum of Compound 1 modified (TL-1) (From 70.0 to $0.0_{\mathrm{fl}}$ and From 4.20 to $1.80_{\mathrm{f}}$ ) (mixture of veraguensin/ galgravin modified)

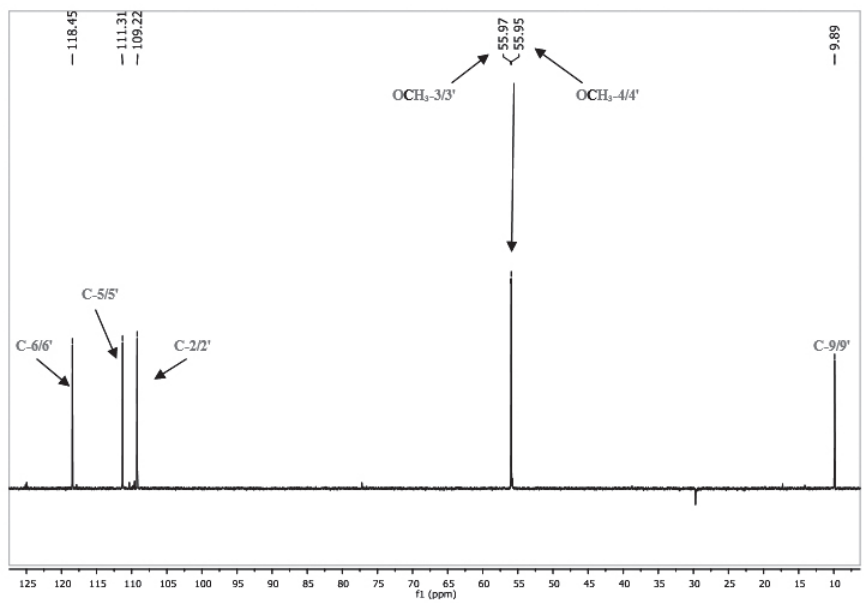

Figure 11. DEPT-135 $5^{\circ}$ Spectrum of Compound 1 modified (TL-1) (mixture of veraguensin/galgravin modified)

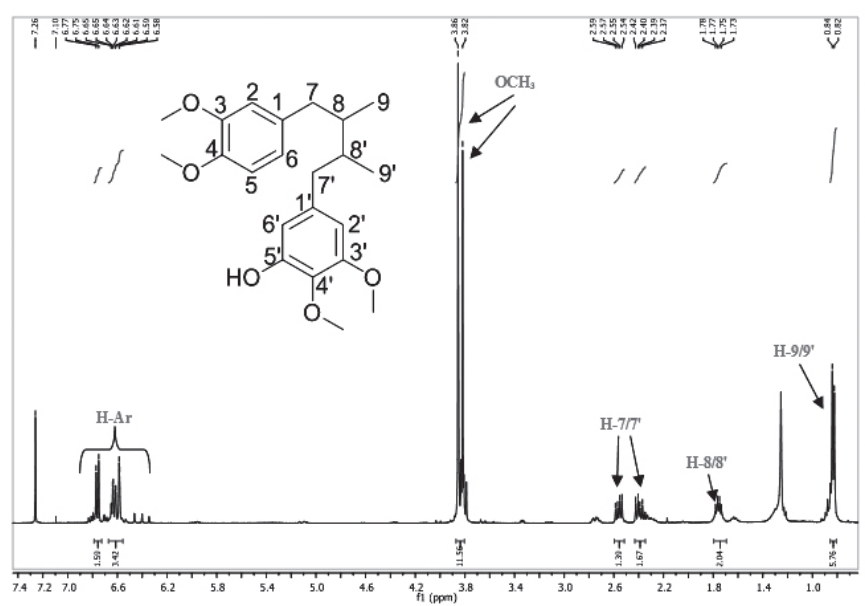

Figure 12. ${ }^{1} \mathrm{H} \mathrm{NMR}\left(400 \mathrm{MHz}, \mathrm{CDCl}_{3}\right)$ Spectrum of Compound 2 (schineolignin $\mathrm{B})$

${ }^{1} \mathrm{H}$ NMR $\left(400 \mathrm{MHz}, \mathrm{CDCl}_{3}\right) \delta 6.76(2 \mathrm{H}, d, J=8.1, \mathrm{ArH}), 6.65(1 \mathrm{H}, d, J$ $=1.9, \mathrm{ArH}), 6.63(1 \mathrm{H}, d d, J=8.1,1.8, \mathrm{ArH}), 6.58(1 \mathrm{H}, d, J=1.8, \mathrm{ArH}), 2.56$ $\left(2 \mathrm{H}, d d, J=13.5,6.8, \mathrm{H}-7 / 7^{\prime}\right), 2.40\left(2 \mathrm{H}, d d, J=13.7,7.8,7 / 7^{\prime}\right), 1.76(2 \mathrm{H}, d d$, $\left.J=12.9,6.5, \mathrm{H}-8 / 8^{\prime}\right), 0.83\left(6 \mathrm{H}, d, J=6.6, \mathrm{H}-9 / 9^{\prime}\right)$.

\section{Acetylation of schineolignin B (2).}

Schineolignin B $2(2,1 \mathrm{mmol})$, in a mixture of acetic anhydride and pyridine $(5 \mathrm{~mL} / 5 \mathrm{~mL})$ was placed in a $50 \mathrm{~mL}$ pear-shaped flask. The mixture was stirred at $100 \mathrm{C}$ for $15 \mathrm{~h}$. Removal of the solvent under reduced pressure afforded a crude mixture, which was extracted with $\mathrm{HCl}$ solution followed by extracted with $\mathrm{NaHCO}_{3}$ solution to give the products, which was purified by column chromatography on silica gel (n-hexane/AcOEt $=8 / 2)$ Sephadex $L H$ 20 in open column chromatography to give 5-(4-(3,4-dimethoxyphenyl)-2,3dimethylbutyl)-2,3-dimethoxyphenyl acetate, $85 \%$ (TL-2) [11].

${ }^{1} \mathrm{H} N M R\left(400 \mathrm{MHz}, \mathrm{CDCl}_{3}\right.$ ) spectral data of Schineolignin B: ${ }^{1} \mathrm{H}$ NMR $\left(400 \mathrm{MHz}, \mathrm{CDCl}_{3}\right) \delta: 6.76(1 \mathrm{H}, d, J=8.1, \mathrm{Ar}-\mathrm{H}), 6.65(1 \mathrm{H}, d, J=1.9, \mathrm{Ar}-\mathrm{H})$, $6.63(1 \mathrm{H}, d d, J=8.1,1.8, \mathrm{Ar}-\mathbf{H}), 6.58(1 \mathrm{H}, d, J=1.8, \mathrm{Ar}-\mathrm{H}), 2.56(2 \mathrm{H}, d d, J=$ $\left.13.5,6.8, \mathrm{H}-7 / 7^{\prime}\right), 2.40\left(2 \mathrm{H}, d d, J=13.7,7.8, \mathrm{H}-7 / 7^{\prime}\right), 1.76(2 \mathrm{H}, d d, J=12.9$, $\left.6.5, \mathrm{H}-8 / 8^{\prime}\right), 0.83(6 \mathrm{H}, d, J=6.6, \mathrm{H}-9 / 9 ')$.

${ }^{1} \mathrm{H} N \mathrm{NR}(400 \mathrm{MHz}, \mathrm{CDCl})$ spectral data of TL-2: $\delta: 6.76-6.57(5 \mathrm{H}, \mathrm{m}$, Ar-H), 3.86-3.81 (12H, $\left.s, 3 \times \mathrm{OCH}_{3}\right), 2.56\left(2 \mathrm{H}, d d, J=13.5,6.7, \mathrm{H}-7 / 7^{\prime}\right), 2.40$ $\left(2 \mathrm{H}, d d, J=13.5,7.8, \mathrm{H}-7 / 7^{\prime}\right), 2.30$ ( $\left.3 \mathrm{H}, s, \mathrm{CH}_{3}-\mathrm{CO}_{2}-\mathrm{Ar}\right), 1.79-1.73(\mathrm{~m}, 2 \mathrm{H}$, $\left.\mathrm{H}-8 / 8^{\prime}\right), 0.83\left(d, J=6.6,6 \mathrm{H}, \mathrm{H}-9 / 9^{\prime}\right)$ (see supporting information, Figure 13). 
Benzylation of meso-dihydroguaiaretic acid and threo-dihydroguaiaretic acid (3).

A mixture of $\mathbf{3}(0.567 \mathrm{mmol})$ and sodium carbonate $(11.4 \mathrm{mmol})$ in dry acetone $(36 \mathrm{ml})$ was heated to reflux for $1 \mathrm{~h}$ under nitrogen. Then, benzyl bromide $(0.63 \mathrm{ml}, 5.67 \mathrm{mmol})$ was added and the mixture was heated under reflux for an additional $3 \mathrm{~h}$. After cooling to room temperature, the reaction mixture was filtered. The filtrate was concentrated and distilled under reduced pressure in a rotary evaporator to remove the excess unreacted benzyl bromide. The residue was chromatographed on silica gel (hexane/AcOEt $=8 / 2$ ) and Sephadex $\mathrm{LH}$-20 in open column chromatography to give 1-(benzyloxy)-4-(4(3,4-dimethoxyphenyl)-2,3-dimethylbutyl)-2-methoxybenzene, 80\% (TL-3) $[12,13]$.

${ }^{1} \mathrm{H} \mathrm{NMR}(400 \mathrm{MHz}, \mathrm{CDCl}$ ) spectral data of meso-dihydroguaiaretic acid and threo-dihydroguaiaretic acid: $\delta: 6.82(d, J=8.0,2 \mathrm{H}), 6.78(d d, J=8.2,2.3$, $2 \mathrm{H}), 6.67(d d, J=8.1,1.9,1 \mathrm{H}), 6.63(d, J=1.8,1 \mathrm{H}), 6.60(d d, J=8.0,1.8,2 \mathrm{H})$, $6.59(d, J=1.9,1 \mathrm{H}), 6.54(d, J=1.8,2 \mathrm{H}), 2.75(d d, J=13.5,5.0,2 \mathrm{H}), 2.54(d d$, $J=13.5,7.1,2 \mathrm{H}), 2.40(d d, J=13.6,7.6,2 \mathrm{H}), 2.30(d d, J=13.5,9.2,1 \mathrm{H}), 1.76$ $(d d, J=13.3,6.7,2 \mathrm{H}), 1.75(d d, J=13.0,6.6,2 \mathrm{H}), 0.85(d d, J=6.6,2.5,6 \mathrm{H})$.

${ }^{1} \mathrm{H} \mathrm{NMR}\left(400 \mathrm{MHz}, \mathrm{CDCl}_{3}\right)$ spectral data of TL-3: $\delta: 7.46(2 \mathrm{H}, d, J=7.3$, $\mathrm{H}-2 " / 2 "$ and 6"/6" $), 7.38(2 \mathrm{H}, t, J=7.4, \mathrm{H}-3$ " $/ 3$ " " and $\mathrm{H}-5 " / 5 "), 7.32(1 \mathrm{H}$ $t, J=7.2, \mathrm{H}-4 " / 4 " ”), 6.81(2 \mathrm{H}, d, J=9.3, \mathrm{Ar}-\mathrm{H}), 6.79(2 \mathrm{H}, d, J=8.3, \mathrm{Ar}-\mathrm{H})$ $6.69(1 \mathrm{H}, d, J=1.6, \mathrm{Ar}-\mathrm{H}), 6.64(1 \mathrm{H}, d d, J=9.4,1.5, \mathrm{Ar}-\mathrm{H}), 6.57(1 \mathrm{H}, d d, J$ $=8.1,1.6, \mathrm{Ar}-\mathrm{H}), 5.14\left(4 \mathrm{H}, s, \mathrm{H}-77^{\prime} / 7^{\prime \prime}\right), 2.75\left(2 \mathrm{H}, d d, J=13.4,4.9, \mathrm{H}-7 / 7^{\prime}\right)$ 2.57 ( $2 \mathrm{H}, d d, J=13.6,6.7, \mathrm{H}^{-7 / 7}$ ') 2.40 (2H, $\left.d d, J=13.6,7.8, \mathrm{H}-7 / 7^{\prime}\right), 2.30$ $\left(2 \mathrm{H}, d d, J=13.4,9.3, \mathrm{H}-7 / 7^{\prime}\right), 0.86\left(3 \mathrm{H}, d, J=7.2, \mathrm{H}-9 / 9^{\prime}\right), 0.84(3 \mathrm{H}, d, J=$ $6.8, \mathrm{H}-9 / 9$ ') (see supporting information, Figure 15).

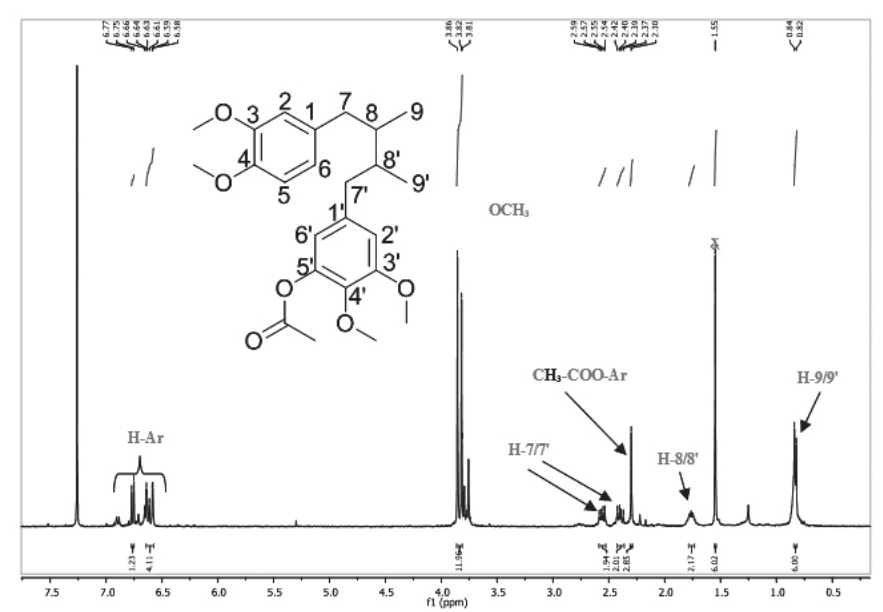

Figure 13. ${ }^{1} \mathrm{H}$ NMR (400 $\left.\mathrm{MHz}, \mathrm{CDCl}_{3}\right)$ Spectrum of Compound 2 modified (TL-2) (schineolignin B modified)

${ }^{1} \mathrm{H}$ NMR (400 MHz, $\left.\mathrm{CDCl}_{3}\right) \delta 6.76-6.57(5 \mathrm{H}, m, \mathrm{ArH}), 3.86-3.81(12 \mathrm{H}$, $\left.s, 3 \times \mathrm{OCH}_{3}\right), 2.56\left(2 \mathrm{H}, d d, J=13.5,6.7, \mathrm{H}-7 / 7^{\prime}\right), 2.40(2 \mathrm{H}, d d, J=13.5,7.8$, $\left.\mathrm{H}-7 / 7^{\prime}\right), 2.30$ (3H, $\left.s, \mathrm{CH}_{3}-\mathrm{CO}\right), 1.79-1.73\left(2 \mathrm{H}, m, \mathrm{H}-8 / 8^{\prime}\right), 0.83(6 \mathrm{H}, d, J=$ 6.6, H-9/9').

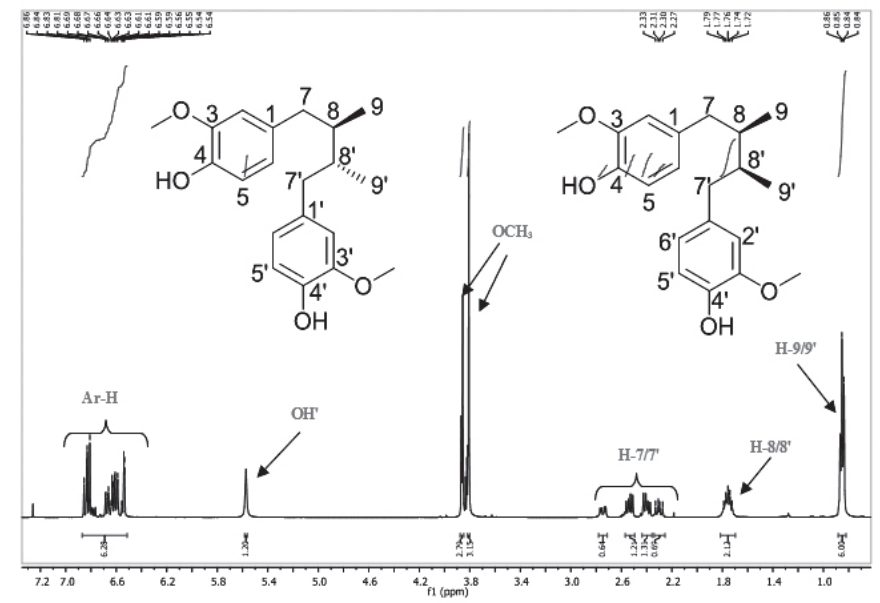

Figure 14. ${ }^{1} \mathrm{H}$ NMR $\left(400 \mathrm{MHz}, \mathrm{CDCl}_{3}\right.$ ) Spectrum of Compound 3 (mixture of meso-dihydroguaiaretic acid and threo-dihydroguaiaretic acid)

${ }^{1} \mathrm{H} \mathrm{NMR}\left(400 \mathrm{MHz}, \mathrm{CDCl}_{3}\right) \delta 6.82(2 \mathrm{H}, d, J=8.0), 6.78(2 \mathrm{H}, d d, J=8.2$, 2.3), $6.67(1 \mathrm{H}, d d, J=8.1,1.9), 6.63(1 \mathrm{H}, d, J=1.8), 6.60(2 \mathrm{H}, d d, J=8.0$ $1.8), 6.59(1 \mathrm{H}, d, J=1.9), 6.54(2 \mathrm{H}, d, J=1.8), 5.44(1 \mathrm{H}, \mathrm{OH}), 2.75(2 \mathrm{H}, d d$ $J=13.5,5.0), 2.54(2 \mathrm{H}, d d, J=13.5,7.1), 2.40(2 \mathrm{H}, d d, J=13.6,7.6), 2.30$ $(2 \mathrm{H}, d d, J=13.5,9.2), 1.76(2 \mathrm{H}, d d, J=13.3,6.7), 1.75(2 \mathrm{H}, d d, J=13.0,6.6)$, $0.85(6 \mathrm{H}, d d, J=6.6,2.5)$

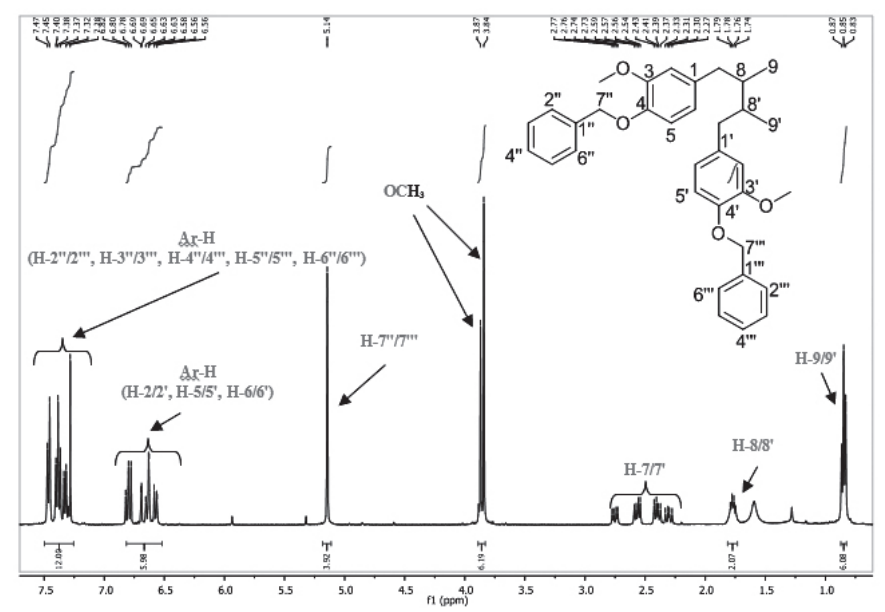

Figure 15. ${ }^{1} \mathrm{H}$ NMR (400 MHz, $\mathrm{CDCl}_{3}$ ) Spectrum of Compound 3 modified (TL-3) (mixture of meso-dihydroguaiaretic acid and threo-dihydroguaiaretic acid modified)

${ }^{1} \mathrm{H}$ NMR (400 MHz, CDCl $){ }^{1} \mathrm{H}$ NMR (400 MHz, $\left.\mathrm{CDCl}_{3}\right) \delta 7.46(2 \mathrm{H}, d$, $J=7.3, \mathrm{H}-2 " / 2 "$, and $6 " / 6 ",), 7.38(2 \mathrm{H}, t, J=7.4, \mathrm{H}-3 " / 3 "$, and $\mathrm{H}-5 " / 5 "$,$) ,$ $7.32(1 \mathrm{H}, t, J=7.2, \mathrm{H}-4 " / 4 "),, 6.81(2 \mathrm{H}, d, J=9.3, \mathrm{Ar}-\mathrm{H}), 6.79(2 \mathrm{H}, d, J=$ 8.3, Ar-H), $6.69(1 \mathrm{H}, d, J=1.6, \mathrm{Ar}-\mathrm{H}), 6.64(1 \mathrm{H}, d d, J=9.4,1.5, \mathrm{Ar}-\mathrm{H}), 6.57$ $(1 \mathrm{H}, d d, J=8.1,1.6, \mathrm{Ar}-\mathrm{H}), 5.14(4 \mathrm{H}, s, \mathrm{H}-7 " / 7 " '), 2.75(2 \mathrm{H}, d d, J=13.4$ $\left.4.9, \mathrm{H}-7 / 7^{\prime}\right), 2.57\left(2 \mathrm{H}, d d, J=13.6,6.7, \mathrm{H}-7 / 7^{\prime}\right), 2.40(2 \mathrm{H}, d d, J=13.6,7.8$, H-7/7'), 2.30 ( $\left.2 \mathrm{H}, d d, J=13.4,9.3, \mathrm{H}-7 / 7^{\prime}\right), 0.86\left(3 \mathrm{H}, d, J=7.2, \mathrm{H}-9 / 9^{\prime}\right), 0.84$ $\left(3 \mathrm{H}, d, J=6.8, \mathrm{H}-9 / 9^{\prime}\right)$.

\section{RESULTS AND DISCUSSION}

Three structural transformation process are presented in this article; and corresponds to the first report of this type of structural modification of lignans isolated from Nectandra species. A direct method was developed for the conversion of compound $\mathbf{1}$ to furan-type lignan. Additionally, the structural transformation of compounds 2 [14] (benzylation); and compound 3 [15] (acetylation). The spectroscopic data comparison between the initial 
and the transformed compound showed formation of derivatives compounds (see supporting information for details). Interestingly, few reports describe dehydrogenation, benzylation or acetylation of natural products isolates; to our knowledge the direct structural transformation of lignan compounds isolated from Nectandra species has yet to be documented.

Comparison of spectroscopic data between the starting material (veraguensin and galgravin) and the product (TL- 1) show the absence some characteristics signals [such as: $4.42(1 \mathrm{H}, d, J=9.3, \mathrm{H}-7), 5.14(1 \mathrm{H}, d, J=8.6$, H-7'), and $\left.4.52\left(2 \mathrm{H}, d, J=6.4, \mathrm{H}-7 / 7^{\prime}\right)\right]$, allow suggest the formation of TL-1.

The compound TL-2 has a signal $2.30\left(3 \mathrm{H}, s, \mathrm{CH}_{3}-\mathrm{CO}\right)$, among others; which it is characteristic of the formation of the product.

The compound TL-3 has a signal 5.14 (4H, $\left.s, \mathrm{H}-7^{\prime \prime} / 7^{\prime \prime}\right)$, among others; which it is characteristic of the formation of the product. Additionally, the compound formed is absent the signal generated by the hydroxyl group $[5.44(1 \mathrm{H}$, $s, \mathrm{OH})]$.

\section{REFERENCES}

1. J. M. Barbosa-Filho, M. Yoshida, O. R. Gottlieb, Phytochemistry. 28, 1991, (1989).

2. L. Chérigo, V. Polanco, E. Ortega-Barria, M. V. Heller, T. L. Capson, L. C. Rios, Nat. Prod. Res. 19, 373, (2005).

3. A. A. da Silva Filho, S. Albuquerque, M. L. e. Silva, M. N. Eberlin, D. M.
Tomazela, J. K. Bastos, J. Nat. Prod., 67, 42, (2004).

4. S. R. Farias-Moreno, A. Arnobio, J. José de Carvalho, A. L. Nascimento, M. O. Timoteo, B. Olej, E. K. Rocha, M. Pereira, M. Bernardo-Filho, L. Querino de Araújo Caldas, Biol. Res. 40, 131, (2007).

5. J. C. Moro, J. B. Fernandes, P. C. Vieira, M. Yoshida, O. R. Gottlieb, H. E. Gottlieb, Phytochemistry, 26, 269, (1987).

6. B. Agius, M. Setzer, S. Stokes, T. Walker, W. Haber, W. Setzer, Int. J. Essen. Oil Ther. 1, 167, (2007).

7. J. G. Rohwer. Lauraceae: Nectandra. Flora Neotropica, Monograph 60, in Flora Neotropica Monograph. vol. 60, T. N. Y. B. Garden, Ed., ed New York, pp. 1-332, 1993.

8. J. Chen, W. Li, H. Yao, J. Xu, Fitoterapia. 103, 231, (2015).

9. M. Saleem, H. J. Kim, M. S. Ali, Y. S. Lee, Nat. Prod. Rep, 22, 696, (2005).

10. L. Dalla Via, E. Uriarte, E. Quezada, A. Dolmella, M. G. Ferlin, O. Gia, J. Med. Chem. 46, 3800, (2003).

11. R. Nakamura, Y. Obora, Y. Ishii, Tetrahedron. 65, 3577, (2009).

12. H. S. P. Rao, S. Senthilkumar, J. Chem. Sci. 113, 191, (2001).

13. L. McMaster, W. Bruner, Ind. Eng. Chem. 28, 505, (1936).

14. M. Miyazawa, H. Kasahara, H. Kameoka, Phytochemistry. 46, 1173, (1997)

15. Y. B. Xue, Y. L. Zhang, J. H. Yang, X. Du, J. X. Pu, W. Zhao, X. N. Li, W. L. Xiao, H. D. Sun, Chem. Pharm. Bull. 58, 1606, (2010)- 